\title{
[ 239 ]
}

\section{VIII.}

\section{E R, S U C H E}

über

das AbJorptionsuermögen der Kohle

vom

Grafen Cari Lud von Morozzo. *)

Ich hatte im Jahre 1783 im Journal de Phyfique zwei Auffätze uber die Abforption der atmofphärifchen Luft und der verfchiedenen Gasarten durch die Kohle bekannt gemacht. " ) Sie wurden in, das Englifche, ins Deutfche und ins Italiänifche aberfetzt, und in mehrere phyfikalifche und chemilche Werke übergetrajen. Van Noordan wiederhohlte meine Verfuche, und erhielt diefelben Refultate; er und $R$ ouppe in Rotterdam fanden, dufs die Kohle, auch nachdem fie erlofchen und erkaltet jft, das Vermögen beibehält, die Gasarten $\mathrm{zu}$ verfchlucken; ${ }^{* *}$ ) auch ich hatte wahr-

*) Zufaminen gezogen, mit Uebergebung mancher veralteiten Meinung, aus dem Journal de Phyf.o t. 57, p. 465 .

d. $H$.

* j Journ. de Phy\%, 17-83, Aur., Nov. S. Lichten. berg's Magazin, B. 2, St. 2, S. 7, u. St. 3, S. 72. d. $H$.

\#**) Vergl, Scherer's allg. Journ. d. Chemie, Th. 3, S. 30o.

d. $H$. 


\section{[ 240 ]}

genommen, dafs nach gänzlichem Erkalten der Kohle noch Gas verfchluckt wurde. Endlich hat van Mons die Abhandlung Rouppe's mit einigen intereffanten Zufätzen bereichert. ") Bei deri allen bleibt indefs noch gar viel über diefen Gegenftand zu unterluchen.

Das Interefle, welches die Phyliker an diefen Verfuchen genonmen haben, beltimmt mich, ihnen auch die vorzulegen, welche ich leit $17 \delta^{8}+$ angeftellt habe, und die jch für einen dritten Auffatz beltimmt hatte. Politilche Unruhen verhinderten mich, fie noch weiter $7 u$ verfolgen, und ich hatte fie gänslich vergeffen, bis fio mir beim Ordnen meiner Papiere im October 1802 wieder in die Hand fiolen.

Verfuch 1: Die Kohle wog in diefem und den beiden folgenden Verfuchen $1 \frac{1}{2}$ Gros, wie in meinen fruhern Verfuchen, und war von Rothbüchenholz, (hatre.) Die Glasröhren, in denen ich die Abforption beobachtete, waren $1 \frac{x}{2}$ Zoll weit und is Zoll lang, und die ganze Einrichtung fo, wie be in meinen beiden frühern Aufätzen be. fchrieben ift. Ich füllte die Röhre über einem Queckfilberapparate mit; fufsfaurem Gas, welches durch concentrirte Schwefelfare aus Flufsfpath ton Maurienne ausgetrieben wurde. Die hinein gebrachte Kchle abforbirte $7^{\prime \prime}$ I"' Gas.

Verfuch 2. Ich brachte darauf die Kohle in Luft, welche ich aus dem faulenden Gewäfer ei-
*) Eben daffelbe, S. 724 .
d. H. 


\section{[ 24I]}

nes Abzugsgrabens aus der Stadt aufgefangen hatte, und die neben dem Kohlenwaflerftoffgas auch kohlenfaures Gas enthielt. Sie ahforbirte davon 6". Diefes ift eine weit gröfsere Abforption, als ich im Wafferftoffgas aus Waffer, Eifen und Schwefelläure erhalten hatte.

Verfuch j. Von dem über'zwei gährenden Weinkufen aufgefangenen Gas abforbirte clie Kohle vom einen 5", vom andern, deffen Kufe in cler Gährung um 5 Tage weiter war, $5^{\prime \prime} 3^{\prime \prime \prime}$, indefs fje vom kohlenfauren Gas gegen 1 "rerfchluckt. Diefes beweilt, dafs jenes Gas, welches im Maximo der Gährung am meiften mephitifch ift, doch immer noch viel atmolphärifche Luft enthält, und defshalb minder tüdulich feyn mufs, als das kohlenfaure Gas. In der That liabe ich gefunden, dals Thiere darin eine Zejt lang leben können. (S. meinen Auff. über die Refpiration im Journ. de Phyf., Aug. 1784.)

Verfuch 4. Der letzte Verfuch, den ich aufgezeichnet finde, wurde mit Kohlen von ver/chiedenen Holzarten in atmofphürifcher Luft angeftellt. Die Kohlen wogen $\frac{1}{2}$ Draclime; die Röhren waren i2"lang und $\frac{1}{2}$ " weit; und man brachte die Kohlen durch das Queckflber, womit fie gefpert waren, hinein. 


\section{[ 242$]$}

Rohle von

Búchenliolz (hêtre)

Weidenhols

Pappelholiz

Hafelnn'sholz (coudrier)

Weinrebe
Abforption Ablorption

binnell 1

Stunde.

" 3 "

$2 \quad 2 \frac{r}{2}$

12 $1 \frac{7}{2}$

112

11 binnen 24

Stunden.

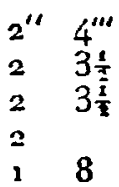

Es ift eine bekannte Erfahrung bei der Bereitung des Schiefspulvers, dafs die Qualität der Kohle auf die Güte des Pulvers Einflufs hat. Sio muls fich möglichlt fchnell entzünden. Dies thut die Kohle eines fehr dichten Holzes nicht, 7. B. von Eichen, Kaftanien, Nufsbaum, Biłchenhols, u. f. f., wefshalb man zur Pulverhereitung die Kohlen von weichem Holze, welches eine viel lockerere Textur hat, vorzieht. Man nimmt kohlen von Hafelnuls, Linden, Weide, Pappel, Erlen oder Faulbaum, und zwar nur von jungen forgfullig ab. gerindeten Zweigen, weil die Rinde und das alte Holz zu wiel erdige Theile [?] enthalten. Bei den Verfuchen zu Effonne fand man, dafs die Kohle von Faulbaum jeder andern vorzuzjehen fey. $\mathrm{Um}$ recht gutes und wirthames Pirfchpulver zu bereiten, verkolılt man auch wohl den holzigen Theil der Hanftengel und deren Splitter. Das mit diefen Kohlen bereitete Pulver habe ich jedes Mahl nach der Pulverprobe als das befte gefunclen. Ich habe felbft eine kleine Probe fehr wirtifamen Pulvers gefehn, dem ftatt der Kohle verbranntes Pa. pier beigemengt ivar. - Die Verkohlung muls 


\section{[ 243 ]}

mit grofser Sorgfalt gemacht werden, wie das in den englifchen Pulverfabriken gefchieht, deren Pulver alles ubrige in Europa an Gitte ubertriffr. Man verkohlt das weifse abgerindete $\mathrm{Hol}$; ị $\mathrm{Cy}$. lindern oder einer Art Ofen aus Metall; diefes ift eine Art von Deftillation, durch welche die Kohle die Eigenfchaft erhält, im Augenblicke entzundet zu werden.

Ich halte es für intereffant, die meiften $\mathrm{Ei}_{\mathrm{i}}$ genfcbaften, welche man bisher an cler Kohle wahrgenommen hat, hier zufammen zu fiellen, damit man uberlegen künne, ob das Analoge in vielen derfelben fich nicht aus einerlei Urfache ableiten laffe.

1. Die Kohle ift einer der fchlechteften Wärmeleiter;

2. dagegen einer der beften Leiter für Electricität und Galvanismus.

3. In der Deftillation giebt fie Wafferftoffgas, [Kohlen - Waifferftoffgas.] Daffelbe gefchieht, wenn man fie glühend in Waffer taucht.

4. Kohlendampf entfärbt viele Pflanzenftoffe [?] und verdirbt die atmolphärifche Luft, fo dafs fie zum Unterhalten der Flamme und zum Ath-. men untauglich wird.

5. Kohle auf einer Schale von Porzellan oder polirtem Glafe dem Thaue ausgefetzt, wird nicht, wie die Metalle, nals, wenn das Porzellan oder das Glas befeuchtet wird. Auch nicht Kohleapulver in einer offenen hölzernen Büchfe, wio ich eben relbft verfucht habe. 


\section{[244]}

6. Kohlenftaub, womit der Boden berleckt ift, verhindert den Schnee, liegen zu bleiben, wie man das an den Orten fehen kann, wo man die Iohlen verkauft.

7. Kohlenpulver verwandelt Eifen in Stahl.

8. Kohlen in Waller gethan, verhindern das Wafler, zu verderben.

9. Kohlenpulver, woruber man verdorbenes Waffer kocht, reinigt es, und nimint demfelben den fauligen Gefchmack, welches, wie van Mons glaubt, nicht durch Desoxygenirung, fondèn durch Oxygenirung, vermöge der verfchluckten atmo. fphärifchen Luft, gefchieht.

10. Koblenpulver dient, Syruppe und anciere Pflanzenextracte zu klüren. Achard bat fich deffelben mit Erfolg zur Raffinirung des Runkelrüben Zuckers bedient.

11. Kohlenftaub noch heifs auf einander gebäuft, entzündet Gch limancmahl von. felbft, wie das in der Pulvermüle zu Elionne der Fall gewefen ift. ${ }^{*}$ )

*) Diefe merkw ürdige Selbftentzündung befchreibt in einem Briefe an Fourcroy der commifjaire en Chef diefer Pulvermühlen, $R$ obin, in den Ann. de Chimie, t. 35, p. 93. , Am z.jften Mai 1799, als eben eine geringe Mlenge pulverifirter Kolle von Faulbaum durchgebeutelt war; äufserte fich beim Oeffnen des Beutelkaftens Hitze, und ein Arbeiter fah Feuer, wie eine Schlange, üher die 


\section{[ 245$]$}

12. Die Kohle hat die Eigenfchaft, den Wein ftein zu entfärhen. ")

13. Man hat fich ihrer mit Erfolg als eines antifeptifchen vilttols in fauligen Krankheiten bedient. [?? Kohlënpulver auf Wunden gefchultet, benimmt ihreen ihren ubeln Geruch.

- Vermuthlich laffen fich alle diefe Erfahrungen dus' einerlei' Urfache erklären. Bis ein ge: fchickter Chemiker und Phyfiker daruber ein helleres Licht verbreitet haben wird, wird es mir erlauht feyn, meine Meinung mitzutheilen. Ich glaube, dafs die Kohle unter allen Körpern am

die Oberfläche der Kohle hinlaufen. Man mach. te Löcher in deri obern Theil des Kafters und gols Warfer hinein, allein die Kohle blieb bren. nend auf der Oberfäche des Walfers fchwimmen, und wurde erft şelofcht, als man fie mit einem befen unter das Wafler hrachte. Im Beutelkaften lag damahls gerade das Produkt meh. rerer Verkohiungen von Faulbaumbol?. Das letztere war erlt den Abend vorher um : Uhr aus dem Ofen geingen, und wie gewöhnlich zum Erfticken der Gluth in ein genau verlchloflenes Behdlenifs gebracht, und darin bis 5 Uhr Morgens, alfo 14 Stunden lang, yelaflen worden. Darauf hatte man es an einem offnen Orte ge. fiebt, urid dann unter Mühlfieinen zu einem Pulver gemahlen. Bei allen diefen Prozelfen hatte den Arbeitern die Kohle dem Gefühl nach fo warm nicht gefchienen, als fie wohl manch. mahl wird, hatte fich auch kein Funkchen Feuer gezeigt, das, wäre es da gewelen, durch Anoal. d. Pbylak. B. 17. St. 2.J. 1804. S. 6. 


\section{[ 246 ]}

meiften Lichtiftoff und Wärmematerie befitzt, Ge tiberall begierig ein[chluckt, und fie, fo zu fagen, mit fich verkörpert. Dafür fcheinen mir folgende Verfuche zu Sprechen.

1. Zwei Thermometer, die mehrere Tage lang im Schatten hingen, hielten einen völlig gleichen Gang. Auf die Kugel des einen legte ich ein ausgehühltes Stuck Buchenkohle; nun zeigte es immerfort 1 bis $1 \frac{\pi}{2}{ }^{\circ} \mathrm{R}$. melur als das andere, fo wohl an Regen - und Nebeltagen als bei bellem Wetter, und in der Kälte wie in mäfsiger Temperatur. Zuletzt fchı̈ängert fich die Kohle mit Feuchtigkeit, und dann verliert lie diefe Eigenfchaft.

die Arbeit Tellft notbwendig hätte mülfen angefacht werden. Die kleinen Kohlenftücke, die beim Sieben zurück behalten waren, hatten an freier Luft gelegen, und fich nicht entzündet. Die Bewegung des Beurels heim Beuteln ift nur mätsig, und das Kohlenpulver war ohne alle fremde Beimifchung. Es fctieint daher gewifs zu feyo, dafs diefes eine Selbftentzündung der Kohle für fich war, veranlalst durch die Hitze, welche das Kohlenpulver, als ein Tehr fchlechter Warmeleiter, noch behaiten hatte. Die Luft war etwas feucht, das Wetter nicht heils, und kein Anzeichen von Gewitter. Vielleicht, dafs durch cine ähnliche Eatzündung manche von den Pulvermiihlen, in welchen dio Kohle zerftampft wird, anfgeflogen ifr." So weit Robin. d.H. *) Viel mehr Eigenfchaften diefer Art findet max angegeben in den Ann., XIII, $103 . \quad$ d. $H$. 


\section{[ 247 ]}

2. Als auf das eineThermometer eine gewöhnliche, auf das andere eine langiam gehrannte Kohle, (welche man defshalb für beffer hält,) gelegt wurde, ftand das letztere Thermometer immer $\frac{x}{2}{ }^{\circ}$ höher als das erfte.

3. Als ider Verfuch mit einer Bachenkohle, die 4 Stunden lang in den Sonnenftrahlen gelegen hatle, náachièn fie bis zur Lufttemperatur wieder herab gekommen war, angeftellt wurde, zeigte das Thermometer, auf dem lie lag, einen grüfsern Wärmegrad, als das andere mit gewöhnlicher Kohle.

4. Dafs es hierbei weder blofs auf eine Hullo gegen Berührung der Luft, noch anf einen fchwarzen Ueberzug der Thermometerkugel ankam, erhellt daraus, dafs, als ich den Verfuch mit Holz oder Bimsfteln, die fchwarz gefärht waren, wiederhohlte, der Effekt ausblieb.

Noch andere felır überzeugende Verfuche, die jch angeftellt habe, behalte ich mir vor, fammt den Inftrumenten, mit denen fie unternommen wurden, in einem eigoen Auffatze zu befchreiben. 